\title{
The Generation R study: a candidate gene study and genome-wide association study (GWAS) on health-related quality of life (HRQOL) of mothers and young children
}

\author{
Hein Raat - Lenie van Rossem • Vincent W. V. Jaddoe • \\ Jeanne M. Landgraf • David Feeny • Henriëtte A. Moll • \\ Albert Hofman · Johan P. Mackenbach
}

Accepted: 10 October 2010/Published online: 28 October 2010

(C) Springer Science+Business Media B.V. 2010

\begin{abstract}
Aim The aim of this paper is to describe the Generation $\mathrm{R}$ study as a template that enables candidate gene study and genome-wide association study regarding health-related quality of life (HRQOL) of mothers and their young children.

Methods Generation R is a population-based prospective cohort study from fetal life onwards in Rotterdam, The Netherlands. Children were born in 2002-2006. Blood from mothers and placenta cord blood were sampled. Mothers' HRQOL was measured 5 times during pregnancy and after birth using SF-12 and EQ-5D. Children's
\end{abstract}

H. Raat $(\bowtie) \cdot$ L. van Rossem · J. P. Mackenbach

Department of Public Health, Erasmus MC, University Medical

Center Rotterdam, P.O. Box 2040, 3000 CA Rotterdam,

The Netherlands

e-mail: h.raat@erasmusmc.nl

L. van Rossem · V. W. V. Jaddoe

Generation R, Erasmus MC, University Medical Center

Rotterdam, Rotterdam, The Netherlands

J. M. Landgraf

HealthActCHQ, Cambridge, MA, USA

D. Feeny

The Center for Health Research,

Kaiser Permanente Northwest, Portland, OR, USA

D. Feeny

Health Utilities Incorporated, Dundas, ON, Canada

H. A. Moll

Department of Pediatrics, Erasmus MC, University Medical Center Rotterdam, Rotterdam, The Netherlands

\section{A. Hofman}

Department of Epidemiology, Erasmus MC, University Medical Center Rotterdam, Rotterdam, The Netherlands
HRQOL was measured 5 times between age 1 and $5 / 6$ years using Infant-Toddler Quality Of Life questionnaire (ITQOL), Health Status Classification System PreSchool (HSCS-PS) and Child Health Questionnaire Parent Form 28 items (CHQ-PF28), respectively.

Results DNA is available for 8,055 mothers and 5,908 children. Genotyping of various candidate genes and a genome-wide association (GWA) scan (Illumina 610K) of child DNA were done. A template for gene-HRQOL analyses is provided. We start with candidate gene study on HRQOL of mothers and children. Gene-environment interaction and interaction with medical indicators of health status will be explored. Next, GWA study on HRQOL will be performed.

Conclusions Gaining insight into the determinants of HRQOL is essential to assisting efforts in health policy and clinical application to improve well-being and health. In the future, it might be possible to complement HRQOL assessments by examinations of genetic markers. Strengths and weaknesses of the Generation R study are discussed.

Keywords Genetic factors - Genome-wide association study (GWAS) · Health-related quality of life .

Mothers · Children

\section{Introduction}

Quality of life may be defined as 'individuals' perceptions of their position in life in the context of the culture and value systems in which they live and in relation to their goals, expectations, standards and concerns (WHO Quality of Life group definition [1]), or as 'physical, social and emotional aspects of well-being that are relevant and important to the individual' [2]. As such, it is a 
multi-dimensional concept. There are validated questionnaires for adults as well as for adolescents, children, infants and toddlers to measure health-related quality of life (HRQOL) [3, 4]. The relevant dimensions and the item content of pediatric quality of life questionnaires differ from those applied in adult questionnaires and vary with developmental age [4]. In young children, it is indispensable to use a proxy rater to complete the HRQOL questionnaire; the proxy rater generally is one of the parents [3].

Health-related quality of life may be studied either in general population samples or in clinical samples of patients. At the general population level, the purpose of such studies is often to monitor the health status and wellbeing; for the purpose of public health policy, it is important to know the determinants of HRQOL in order to develop public health policies $[5,6]$. With regard to patients with a certain medical condition, clinicians assess the impact of the condition on HRQOL and the factors that determine quality of life in order to find ways for improving the patients' well-being [7-10]. In the case of intensive or new therapies, clinicians monitor the impact of therapy on HRQOL [11-13]. In clinical trials, HRQOL is an outcome measure, complementary to clinical measures and mortality [3].

While in general population studies and in clinical studies, the major determinants of health-related quality of life have been identified, i.e., the severity and duration of symptoms, the effects of specific medical interventions, socio-demographic and -economic characteristics such as gender, age, education and income level, as well as certain psychosocial aspects such as social support and stressful life events, only little is known about potential genetic aspects $[14,15]$. The GENEQOL consortium was established to investigate the genetic disposition of HRQOL and other related patient-reported outcomes in addition to the known determinants of quality of life [14, 15]. The consortium hypothesizes that since concepts that are closely related to HRQOL, such as negative and positive psychological affect, major depressive disorder, poor self-rated physical health, pain and fatigue have genetic components [15-19], it is likely that genes can be found that are associated with HRQOL.

The ultimate goal of gaining insight into the genetic disposition of HRQOL is to find better ways to improve general health, well-being and quality of life by targeting subgroups that may benefit from specific interventions. The first step is a candidate gene approach, which includes empirical research across multiple datasets to establish whether a proposed list of potentially relevant genetic factors reveals genes that are statistically significantly associated with specific domains of HRQOL [15]. In a second phase, as suggested by the GENEQOL consortium, genome-wide association studies in large samples are needed to identify potential genetic factors that affect HRQOL. Replication of results in independent samples is required.

The Generation R study, a prospective population-based cohort study that was designed to identify early environmental and genetic causes of normal and abnormal growth, development and health from fetal life onwards, is appropriate to contribute to the study of genetic factors related to HRQOL of mothers and children. The study has previously been described in detail [20-22]. The focus of this paper is to discuss how HRQOL data can be integrated into genetic research. We describe the Generation $\mathrm{R}$ study as an example of a cohort study enabling candidate gene studies and genome-wide association studies of HRQOL of both mothers and their young children.

\section{Methods}

Design and participants

The Generation R study was conducted in Rotterdam, the Netherlands, in a multi-ethnic metropolitan area. Pregnant women were informed about the study and invited to provide written informed consent by their prenatal care provider during the first visit in pregnancy. The study area includes approximately 350.000 inhabitants of circa 150 different ethnicities [23]. The Generation $\mathrm{R}$ study was conducted in accordance with the guidelines proposed in the World Medical Association Declaration of Helsinki and has been approved by the Medical Ethical Committee of the Erasmus MC, University Medical Center Rotterdam.

Mothers in the study area with a delivery date between April 2002 and January 2006 were eligible for participation. The majority of mothers were enrolled during early pregnancy (gestational age $<18$ weeks). Fathers were invited to participate but did not complete HRQOL questionnaires.

Assessments during pregnancy, including questionnaires with regard to maternal HRQOL, took place in early pregnancy (gestational age $<18$ weeks), mid-pregnancy (gestational age 18-25 weeks) and late pregnancy (gestational age $>25$ weeks). At ages 2, 6, 12, 18, 24, 30, 36 and 48 months, parents (most often mothers) completed questionnaires for themselves and their infant. Furthermore, data were collected during routine visits to the preventive child health centers at ages 1, 2, 3, 4, 6, 11, 14, 18, 24, 30, 36 and 45 months, respectively. At age 5/6 years, data were collected during a visit of the parent and child to the Erasmus Medical Center using hands-on measurements and standardized parent-completed questionnaires. 
Health-related quality of life of the mothers

In early pregnancy (gestational age $<18$ weeks), we administered the SF-12, 3 additional items of the SF-36 mental health scale [24-27], the EQ-5D and a visual analogue scale (VAS) on general health of the mother [24, 28, 29]. At mid-pregnancy and at late pregnancy, the SF-12 and the entire SF-36 mental health scale were administered again. Two months after the child's birth, all measures were repeated, but at the 6-month-postbirth assessment, only the SF-12 was administered.

The SF-12, and even more the EQ-5D, may show ceiling effects in general population samples; in subgroups with ill health, especially the SF-12 may be subject to floor effects [30]. Thus, during the analytic phase of the project, it will be essential to determine the range of health status outcomes for both the SF-12 and the EQ-5D. In addition to the SF-12 summary score formulas proposed by Ware et al. that are calculated by adding up positive weights and subtracting negative weights, we will also apply the RAND-12 formulas to calculate summary scores that only use positive weights, since some studies have shown that the SF-12 physical and mental domains may interact [31].

Health-related quality of life of the children

Age 12 months: At age 12 months, we applied the InfantToddler Quality of Life (ITQOL) questionnaire. This is a parent-completed generic HRQOL measure for infants and toddlers aged 2 months up to 5 years of age [32-35]. The ITQOL consists of 103 items with four, five or six Likerttype response options, divided over ten multi-item scales and two single-item scales. The ITQOL can be considered a 'health profile measure' [2, 4]. The ITQOL includes scales on physical and psychosocial functioning of the child, as well as on the impact of child health and disease on parental and family functioning.

Age 24 months: To reduce respondent burden and in the absence of a short form of the ITQOL at the time of the study, a factor analytic approach using a prior general population sample [33] was applied to reduce the number of items to be fielded at the follow-up at age 24 months in the Generation R study; 55 items that captured all ITQOL concepts were retained for the purpose of this study only. Before using this ad-hoc short form in future analyses, it is necessary to assess whether the revised version represents the original full length version of the ITQOL and a future final short form of the ITQOL; in the Generation R sample (at age 12 months and age 24 months) and in other studies in which the ITQOL was applied, we propose to evaluate how well the new scorings represent the original scores.

Age 36 months: At the child age of 36 months, we applied a 'preference-based measure' of HRQOL, [2, 4], the
Health Status Classification System for preschool children (HSCS-PS), suitable for children aged 2.5-5 years. The HSCS-PS has 12 dimensions and 3-5 levels per dimension [4, 36-38]. For the purpose of the Generation R study, the parent questionnaire that can be used to classify children according to the HSCS-PS was translated into Dutch following international standards including four forward and two independent backward translations [39].

Age 48 months and age 5/6 years: At both the child age of 48 months and at age 5/6 years, we applied a 'health profile measure', i.e., the Child Health Questionnaire Parent Form with 28 items (CHQ-PF28), which has been developed for ages 5 and older [5, 40-42]. In the Netherlands, the CHQ-PF has been validated for children aged 4 years and older [5]. Like the ITQOL, it covers physical and psychosocial aspects. The CHQ-PF28 permits the calculation of a physical and a psychosocial summary score.

DNA of the mothers and children

Maternal blood samples were collected in early (approximately $35 \mathrm{ml}$ ) and mid-pregnancy (approximately $20 \mathrm{ml}$ ) during the visit to one of the dedicated research centers. Directly after delivery, midwives or obstetricians collected $30-\mathrm{ml}$ cord blood from the umbilical vein. The samples were transported on average within $2 \mathrm{~h}$ to the Generation $\mathrm{R}$ laboratory. Ten-ml EDTA tube per mother and child was provided for DNA extraction; these samples were stored at Erasmus MC, University Medical Center Rotterdam at $-80^{\circ} \mathrm{C}[21]$.

\section{Information on health status and determinants of health}

In the Generation $\mathrm{R}$ study, information has been gathered on the health status of mothers during pregnancy and the postpartum period using 3 prenatal questionnaires and questionnaires 2 and 6 months postpartum with regard to the presence of chronic conditions, symptoms and use of medical services, and using medical information form the patient files and the birth record of the midwife, obstetrician and hospital. During pregnancy, the mothers visited our study center three times and had standardized measures of height, weight, blood pressure and fetal ultrasounds; urine and blood samples were collected [20-22].

The three fetal ultrasounds also provide information on the fetal growth of the children. Until age 6 years, parents complete 10 paper questionnaires including health-related items with regard to their child, including items on symptoms, periods of fever and infections, chronic conditions and use of medical services [20-22]. Data with regard to the growth, development and health status of the children were collected during free routine visits to the 
preventive child health centers, up to 13 preventive health visits until age 6 years. At age 5/6 years, the children (and their parents) are invited to visit our study center in the children's hospital for measurements of growth, body composition and risk factors for cardiovascular disease including a full-body DEXA scan, sonographic measurement of abdominal fat, blood pressure measurement, sonographic measurement of thickness and stiffness of carotid artery wall, assessment of blood cholesterol and lipid profiles. Furthermore, lung function is being assessed, as well as cognitive, mental and behavioral development [20-22].

In the 16 prenatal and postnatal questionnaires for mothers/parents, items are included that assess environmental characteristics. This includes indicators of prenatal and postnatal familial stress, parenting styles and parenting practices in the family, and characteristics of the home and neighborhood environment. Observation of the home environment using a standardized checklist has been done during a home visit at child age 3 months. The home address (postal code and house address) is used to link the Generation $\mathrm{R}$ data to municipal data on the environment including traffic density, environmental noise, air quality, housing quality and social characteristics of the neighborhood [20-22].

\section{Results}

\section{Participants}

Of the eligible mothers, $61 \%$ provided informed consent in the first phase of the study (i.e., pregnancy until birth) [20]. In total, 9,778 mothers were enrolled in the study. Of these mothers, $91 \%$ ( $n=8,880)$ were enrolled during pregnancy. The response rates for the questionnaires during pregnancy and after birth ranged from $60-70 \%$. Mean age of the mother at enrollment was 29.7 (SD 5.3). Parity was 0 for $55 \%, 1$ for $31 \%$ and $>=2$ for $14 \%$ of the mothers in the study. Of the participating mothers, $58 \%$ were Dutch, $9 \%$ Surinamese, $7 \%$ Moroccan, 9\% Turkish, $4 \%$ were of the Dutch Antilles, 4\% Cape Verdian and 9\% of other ethnic background. Forty-two percent of the participating mothers had higher vocational training and/or university-level education [21].

The 9,778 mothers who were enrolled in the study gave birth to 9,745 known live born children. The follow-up until age 48 months was restricted to the catchment's area of the participating preventive child health centers; 1,163 children lived outside this area and were not eligible for follow-up in the first 4 years after birth. After birth, informed consent was renewed; 689 (8\%) mothers did not give consent to the postnatal phase of the study, leaving
7,893 children for the postnatal follow-up until the age of 4 years $[21,22]$.

\section{DNA}

Of the eligible mothers, blood samples for DNA extraction were available for $91 \%(8,055)$. Of the 8,821 eligible children, cord blood samples were obtained for $67 \%$ $(5,908)$. The relative large number of missing cases for cord blood was due to logistical constraints during delivery [21].

It was examined if mothers with and without DNA availability differ with regard to socio-demographic characteristics and birth characteristics. Only small differences between mothers and children with and without DNA availability were observed; the single exception being that children without DNA availability more often had low birth weight $(<2,500 \mathrm{~g} 10.8 \%$, respectively, $3.3 \%)$ and a lower gestational age $(<37$ weeks $11.2 \%$, respectively, $3.9 \%)$ relative to those with available DNA [21].

Child DNA extraction was conducted manually using the Qiagen FlexiGene Kit (Qiagen Hilden, Germany). DNA extraction from 5-ml whole blood samples from the mothers and partners was performed by a Hamilton STAR multi-channel robot using AGOWA magnetic bead technology (at 72 samples per run). Extracted DNA is automatically collected in stock tubes, but DNA of each subject can be individually addressed. All genotyping studies with Generation R DNA material were performed in-house at the genotyping facility associated with Erasmus Medical Center [21].

Candidate gene studies in mothers and children:

a template for data analysis

Table 1 outlines our template for data analysis. We propose to start with assessing the associations with HRQOL outcomes of the (updated) list of candidate genes that was prepared by the GENEQOL consortium based on a literature review regarding genetic factors relating to concepts that are related to HRQOL, i.e., negative and positive psychological affect, self-rated physical health, pain and fatigue [15]. We will thus start with candidate gene studies on HRQOL of both mothers and young children. An advantage of the candidate gene approach is that with single nucleotide polymorphisms (SNPs) with a frequency of at least $5 \%$, one can expect adequate power to assess associations with HRQOL outcomes.

Within the Generation $\mathrm{R}$ study, genotyping of both mother and child DNA has been performed for various specific candidate genes relevant for obesity, diabetes mellitus type 2 , asthma, atopic dermatitis, cortisol secretion and child psychiatric conditions. Other candidate genes that 
Table 1 A template for data analysis in candidate gene studies of HRQOL in mothers and children

\begin{tabular}{ll}
\hline Parameter & Action step \\
\hline Theoretical model & Specify pathways influencing HRQOL \\
Candidate genes & Utilize GENEQOL Consortium list based on literature review regarding \\
& genetic factors relating to HRQOL concepts \\
Gtatistical modeling & Growth models for longitudinal data \\
& General linear models to evaluate associations among potential \\
& determinants and QOL trajectories \\
Foci & HRQOL health status in pathway between genetics determinants and \\
& Gene-environment interactions \\
& Simple sequence changes and their association with HRQOL \\
Copy number variants and their association with HRQOL
\end{tabular}

are hypothesized to be involved in pathways influencing HRQOL may be assessed as well with regard to either mothers or children, or both.

Since the HRQOL outcomes were measured repeatedly, growth models may be applied to evaluate distinct trajectories of quality of life scores over time; General Linear Models may be applied to evaluate associations between potential determinants and trajectories of quality of life scores. In addition, the association between potential determinants and change in quality of life scores over time can be evaluated using longitudinal multilevel analysis. This type of analysis takes account of the correlation between repeated measures on the same subject and allows for incomplete outcome data [43].

The role of health status in the pathway between genetic determinants and HRQOL can also be evaluated. For example, medical conditions such as respiratory disease, or symptoms such as pain, are likely to affect HRQOL. Certain genetic factors may influence HRQOL indirectly through their effect on health status. Gene-environment interactions will also be explored. In addition to simple sequence variations, we can evaluate whether changes in copy number of a small part of the genome, so called copy number variants (CNVs), are associated with HRQOL outcomes [15].

Genome-wide association (GWA) studies

The relatively novel strategy of genome-wide association (GWA) studies is now feasible to determine important common genetic variants that contribute to the explanation of variance in relevant phenotypes (such as high or low HRQOL) [21, 44]. A genome-wide scan (Illumina $610 \mathrm{~K}$ platform) of child DNA was taken. A genome-wide scan of mother DNA has not been scheduled yet.

With regard to child DNA, a multi-stage approach is being prepared for genome-wide association studies on prioritized child phenotypes of interest including child HRQOL. The research strategy will benefit from the infrastructure used in the GWA analyses in the Rotterdam Study, a population-based cohort study in about 15,000 Rotterdam adults [45]. First, all SNPs with a $P$ value $<10^{-7}$ will be selected. Second, these SNPs will be replicated in (where possible) other cohorts that have data on the same self-reported and parent-reported outcomes and DNA. In Generation R, genetic associations will be analyzed in strata of ethnicity and (pooled) as whole cohort.

The Generation $\mathrm{R}$ study groups continue to cooperate with investigators conducting similar child and birth cohort studies that include DNA and data on the same or similar outcomes. Examples include the Avon Longitudinal Study of Parents and Children (ALSPAC) in Bristol, UK, the Danish National Birth Cohort Study, the Norwegian Mother and Child Cohort Study, the Southampton Women's Survey (SWS) in Southampton, UK and The National Children's Study in the USA [21].

\section{Discussion}

It is plausible that genetic factors exist that affect HRQOL of mothers and children. Genetic factors may have a direct effect on HRQOL, but it is also possible that genetic factors influence health status (e.g., disease, severity of symptoms) and hence affect HRQOL. Also, genetic factors may interact with environmental factors. This is an illustration of the extended model of Wilson and Cleary, proposed by Sprangers et al., that shows how molecular and genetic factors influence pathophysiology, symptom status, functional status, general health perceptions and HRQOL [46]. This model also shows how both individual characteristics, including molecular and genetic factors, and environmental characteristics affect each of these steps from pathophysiology to HRQOL [46].

Since the Generation R cohort, which is derived from a general population sample, includes a large majority of healthy mothers and children, with regard to pathophysiologic processes, the focus will be on prevalent conditions 
and symptoms such as those related to asthma, allergies, recurrent pain, or common emotional and behavioral disorders. Lubetkin et al. have shown how socio-demographic factors such as gender, age, education level, ethnic background and income level as well as the presence of common chronic conditions each independently affect HRQOL scores in an adult general population sample [47]. Von Rueden et al. showed that there are similar associations between socio-economic factors and childhood HRQOL [14]. The current study will add whether genetic factors can be found that independently contribute to HRQOL scores of both mothers and children, in addition to currently known determinants. It will be possible to explore whether certain genetic factors interact with socio-demographic determinants or common chronic conditions with regard to the impact on HRQOL.

Until now, there have been very few empirical studies using either a candidate gene approach or a genome-wide association approach to assess the genetic basis of HRQOL. The GENEQOL consortium has proposed a research agenda to provide more insight into the genetic disposition of patient-reported quality of life outcomes. This will require a multi-disciplinary approach. This paper demonstrates that the Generation R study with 9,778 participants in the prenatal phase and 7,893 participants in the postnatal phase can meaningfully contribute to both candidate gene studies of mothers' and children's HRQOL and genome-wide association studies of children's HRQOL.

Since the Generation R study is a longitudinal study, it will be possible to explore whether genetic factors, or genetic factors in interaction with environmental factors, influence trajectories of HRQOL over time. For this purpose, we will first describe the presence of distinct trajectories of HRQOL over time of both mothers (during pregnancy and after delivery) and children (from birth until age 5/6 years), especially in the context of specific (common) medical conditions such as asthma or allergies. Secondly, we will evaluate whether genetic and environmental factors are associated with specific trajectories.

Strengths and weaknesses

The Generation $\mathrm{R}$ study has relatively many participants. However, specific analyses will require available quality of life data as well as data on (child) DNA, reducing the numbers in the actual analyses. It is an advantage that Generation R included a multi-ethnic population; yet for analyses in specific (non-Dutch) ethnic strata, the numbers are relatively low. This pleads for collaborations with other cohorts.

Although overall participation in Generation $\mathrm{R}$ was relatively high $(60 \%)$, it turned out that participants in the study and in separate measurements are relatively higher educated, more often of Dutch origin, and more healthy [20-22]; this needs attention when the associations between potential determinants and HRQOL outcomes are different in the subgroup non-participants compared to the subgroup participants.

Health-related quality of life of both mothers and children was measured repeatedly using widely used validated instruments. However, attention should be given in the analyses to the potential presence of differential item functioning by ethnicity (or gender among children) in this multi-ethnic cohort since this might affect the associations that may arise.

The parents, generally the mothers, completed the questionnaires on the children's HRQOL. Parent characteristics may influence parents' reports on their child's quality of life $[48,49]$, which makes evaluation of the associations between genetic factors and children's HRQOL more complex. In the future, when the children grow older, selfreports on children's quality of life may be collected.

In the Generation R study, a large number of medical, psychological and environmental co-variates have been carefully documented, enabling evaluation of the role of health status in the pathway between genetic factors and HRQOL, and the exploration of gene-environment interactions. However, theoretical models should guide the analyses and attention should be given to the possibility of chance findings given multiple outcomes and multiple determinants; results need replication in other cohorts.

\section{Conclusion}

Gaining insight into the determinants of HRQOL is essential to assisting efforts in health policy and clinical application to improve well-being and health. In the future, it might be possible to complement HRQOL assessments by examinations of genetic markers. This might be especially important in individuals with substantially reduced HRQOL measurement scores that do not correspond with a favorable health and life situation and thus cannot be explained by the classical HRQOL determinants. Specific medical interventions to enhance genetically determined low HRQOL of course should be viewed extremely careful. Beforehand any of such medical interventions could be considered, it should be considered and examined how far such genetic disposition can be compensated by strengthening other resources of quality of life as strong social support, social competence and various personal coping strategies. Specific cognitive interventional or educational approaches could also focus on training people how to cope with such a genetic determinant.

It is now feasible to study the association between candidate genes and HRQOL outcomes. This can occur in 
cohort studies such as the Generation $\mathrm{R}$ study, which is an example of a large birth cohort study with extensive data with regard to health, determinants of health and HRQOL of both mothers and children. As a second step, genome-wide association studies may be performed to find genetic variants related to HRQOL outcomes. In order to proceed efficiently with genome-wide association studies on HRQOL, consensus regarding relevant quality of life concepts is needed as well as collaboration between research centers.

Acknowledgments The Generation R Study is conducted by the Erasmus Medical Center Rotterdam in close collaboration with the Faculty of Social Sciences of the Erasmus University Rotterdam, the Municipal Health Service Rotterdam area, the Rotterdam Homecare Foundation and the Stichting Trombosedienst \& Artsenlaboratorium Rijnmond (STAR), Rotterdam. We gratefully acknowledge the contribution of general practitioners, hospitals, midwives and pharmacies in Rotterdam.

Conflict of interest It should be noted that David Feeny has a proprietary interest in Health Utilities Incorporated, Dundas, Ontario, Canada. HUInc. distributes copyrighted Health Utilities Index (HUI) materials and provides methodological advice on the use of HUI.It should be noted that Jeanne M. Landgraf is affiliated with HealthActCHQ, Cambridge, MA, USA. HealthActCHQ distributes copyrighted Infant-Toddler Quality of Life (ITQOL) questionnaire and Child Health Questionnaire (CHQ) materials and provides methodological advice on the use of ITQOL and CHQ.

\section{References}

1. The WHOQOL Group. (1994). Development of the WHOQOL: Rationale and current status. International Journal of Mental Health, 23(3), 24-56.

2. Connolly, M. A., \& Johnson, J. A. (1999). Measuring quality of life in paediatric patients. Pharmacoeconomics, 16(6), 605-625.

3. Eiser, C., \& Morse, R. (2001). Quality-of-life measures in chronic diseases of childhood. Health Technology Assessment, 5(4), $1-157$.

4. Raat, H., Mohangoo, A. D., \& Grootenhuis, M. A. (2006). Pediatric health-related quality of life questionnaires in clinical trials. Current opinion in Allergy and Clinical Immunology, 6(3), 180-185.

5. Raat, H., Botterweck, A. M., Landgraf, J. M., Hoogeveen, W. C., \& Essink-Bot, M. L. (2005). Reliability and validity of the short form of the child health questionnaire for parents (CHQ-PF28) in large random school based and general population samples. Journal of Epidemiology and Community Health, 59(1), 75-82.

6. Mangunkusumo, R. M. (2006). E-health4Uth, Integrating monitoring and prevention in youth health care. Thesis Erasmus MC, University Medical Center Rotterdam.

7. Bruijn, J., Arts, W. F., Duivenvoorden, H., Dijkstra, N., Raat, H., \& Passchier, J. (2009). Quality of life in children with primary headache in a general hospital. Cephalalgia,. doi:10.1111/ j1468-29822008.01774x.

8. Peetsold, M. G., Hofman, V. E., Huisman, J., Heij, H. A., Raat, H., \& Gemke, R. J. B. J. (2009). Psychological outcome and health-related quality of life in children 6-18 years old born with a congenital diaphragmatic hernia. Archives of Disease in Childhood, 94, 834-840.
9. Mohangoo, A. D., de Koning, H. J., Mangunkusumo, R. T., \& Raat, H. (2007). Health-related quality of life in adolescents with wheezing attacks. Journal of Adolescent Health, 41(5), 464-471.

10. Oostenbrink, R., Spong, K., de Goede-Bolder, A., Landgraf, J. M., Raat, H., \& Moll, H. A. (2007). Parental reports of healthrelated quality of life in young children with eurofibromatosis type 1: Influence of condition specific determinants. Journal of Pediatrics, 151(2), 182-1866. 6 e1-2.

11. Bannink, E. M. N., Raat, H., Mulder, P. G. H., \& Muinck KeizerSchrama, S. M. P. F. (2006). Quality of life after growth hormone therapy and induced puberty in women with turner syndrome. Journal of Pediatrics, 148(1), 95-101.

12. Buysse, C. P. M., Raat, H., Hazelzet, J. A., Hop, W. C. J., Maliepaard, M., \& Joosten, K. F. M. (2008). Surviving meningococcal septic shock: health consequences and quality of life in children and their parents up to 2 years after PICU discharge. Critical Care Medicine, 36(2), 596-602.

13. Prince, F. H. M., Geerdink, L. M., Borsboom, G. J. J. M., Twilt, M., van Rossum, M. A. J., Hoppenreijs, E. P. A. H., et al. (2009). Major improvements in health-related quality of life during the use of etanercept in patients with previously refractory juvenile idiopathic arthritis. Annals of the Rheumatic Diseases, 69, $138-142$.

14. von Rueden, U., Gosch, A., Rajmil, L., Bisegger, C., \& RavensSieberer, U. (2006). Socioeconomic determinants of health related quality of life in childhood and adolescence: results from a European study. Journal of Epidemiology and Community Health, 60(2), 130-135.

15. Sprangers, M. A. G., Sloan, J. A., Veenhoven, R., et al. (2009). The Establishment of the GENEQOL Consortium to Investigate the Genetic Disposition of Patient-Reported Quality-of-Life Outcomes. Twin Research and Human Genetics, 12(3), 301-311.

16. López-León, S., Janssens, A. C. J. W., Gonzalez-Zuloeta Ladd, A. M., Del-Favoro, J., Claes, S. J., Oostra, B. A., et al. (2008). Meta-analyses of genetic studies on major depressive disorder. Molecular Psychiatry, 13, 772-785.

17. Singh, R., Kølvraa, S., Bross, P., Gregersen, N., Andersen Nex $\varnothing$, B., Frederiksen, H., et al. (2004). Association between low selfrated health and heterozygosity for-110A \&gt; C polymorphism in the promoter region of HSP70-1 in aged Danish twins. Biogerontology, 5, 169-176.

18. Kim, H., Ramsay, E., Lee, H., Wahl, S., \& Dionne, R. A. (2009). Genome-wide association study of acute postsurgical pain in humans. Pharmacogenomics, 10, 171-179.

19. Collado-Hidalgo, A., Bower, J. E., Ganz, P. A., Irwin, M. R., \& Cole, S. W. (2008). Cytokine gene polymorphisms and fatigue in breast cancer survivors: Early findings. Brain, Behavior, and Immunity, 22, 1197-1200.

20. Jaddoe, V. W. V., Mackenbach, J. P., Moll, H. A., Steegers, E. A. P., Tiemeier, H., Verhulst, F. C., et al. (2006). The generation R study: Design and cohort profile. European Journal of Epidemiology, 21, 475-484.

21. Jaddoe, V. W., Bakker, R., van Duijn, C. M., et al. (2007). The generation $\mathrm{R}$ study biobank: A resource for epidemiological studies in children and their parents. European Journal of Epidemiology, 22(12), 917-923.

22. Jaddoe, V. W., van Duijn, C. M., van der Heijden, A. J., et al. (2008). The generation R study: Design and cohort update until the age of 4 years. European Journal of Epidemiology, 23(12), 801-811.

23. Center for research and statistics, Rotterdam (COS); http://www. cos.rotterdam.nl; 2005.

24. Essink-Bot, M. L., Krabbe, P. F., Bonsel, G. J., \& Aaronson, N. K. (1997). An empirical comparison of four generic health status measures. The nottingham health profile, the medical outcomes study 36-item short-form health survey, the COOP/WONCA 
charts, and the EuroQol instrument. Medical Care, 35(5), 522-537.

25. Ware, J. E., Keller, S. D., Gandek, B., Brazier, J. E., \& Sullivan, M. (1995). Evaluating translations of health status questionnaires. Methods from the IQOLA project. International quality of life assessment. Int J Technol Assess Health Care, 11(3), 525-551.

26. Ware, J. E., Gandek, B., Kosinski, M., Aaronson, N. K., Apolone, G., Brazier, J., et al. (1998). The equivalence of SF-36 summary health scores estimated using standard and country-specific algorithms in 10 countries: Results from the IQOLA Project. International quality of life assessment. Journal of Clinical Epidemiology, 51(11), 1167-1170.

27. Ware, J. E., Kosinski, M., \& Keller, S. D. (1996). A 12-Item short-form health survey: Construction of scales and preliminary tests of reliability and validity. Medical Care, 34(3), 220-233.

28. Johnson, J. A., \& Coons, S. J. (1998). Comparison of the EQ-5D and SF-12 in an adult US sample. Quality of Life Research, 7, 155-166.

29. Raat, H., Bonsel, G. J., Hoogeveen, W. C., \& Essink-Bot, M. L. (2004). Feasibility and reliability of a mailed questionnaire to obtain visual analogue scale valuations for health states defined by the Health Utilities Index Mark 3. Medical Care, 42(1), 13-18.

30. Fryback, D. G., Cross Dunham, N., Palta, M., Hanmer, J., Buechner, J., Cherepanov, D., et al. (2007). US norms for six generic health-related quality of life indexes from the national health measurement study. Medical Care, 45(12), 1162-1170.

31. Johnson, J. A., Jeffrey, A., \& Maddigan, S. L. (2004). Performance of the RAND-12 and SF- 12 Summary Scores in Type-2 Diabetes. Quality of Life Research, 13(2), 449-456.

32. Landgraf, J. M. (1994). The infant-toddler child health questionnaire: Conceptual framework, logic content, and preliminary psychometric results. Boston: Health Act.

33. Raat, H., Landgraf, J. M., Oostenbrink, R., Moll, H. A., \& EssinkBot, M. L. (2007). Reliability and validity of the Infant and Toddler Quality of Life Questionnaire (ITQOL) in a general population and respiratory disease sample. Quality of Life Research, 16(3), 445-460.

34. Klassen, A. F., Landgraf, J. M., Lee, S. K., Barer, M., Raina, P., Chan, H. W., et al. (2003). Health related quality of life in 3 and 4 year old children and their parents: preliminary findings about a new questionnaire. Health Qual Life Outcomes, 1, 81.

35. Klassen, A. F., Lee, S. K., Raina, P., Chan, H. W., Matthew, D., \& Brabyn, D. (2004). Health status and health-related quality of life in a population-based sample of neonatal intensive care unit graduates. Pediatrics, 113(3 Pt 1), 594-600.

36. Nathan, P. C., Furlong, W., DePauw, S., Horsman, J., van Schaik, C., Rolland, M., et al. (2004). Health status of young children during various phases of therapy for advanced neuroblastoma. Pediatric Blood \& Cancer, 43(6), 659-667.
37. Saigal, S., Rosenbaum, P., Stoskopf, B., et al. (2005). Development, reliability and validity of a new measure of overall health for pre-school children. Quality of Life Research, 14, 243-257.

38. Synnes, A. R., Lefebvre, F., \& Cake, H. A. (2006). Current status of neonatal follow-up in Canada. Paediatr Child Health, 11(5), 271-274.

39. Guillemin, F., Bombardier, C., \& Beaton, D. (1993). Cross-cultural adaptation of health- related quality of life measures: literature review and proposed guidelines. Journal of Clinical Epidemiology, 46(12), 1417-1432.

40. Landgraf, J. M., Abetz, L., \& Ware, J. E. (1996). The CHQ user's manual. Boston: The Health Institute, New England Medical Center.

41. Gorelick, M. H., Scribano, P. V., Stevens, M. W., \& Schultz, T. R. (2003). Construct validity and responsiveness of the child health questionnaire in children with acute asthma. Annals of Allergy, Asthma \& Immunology, 90(6), 622-628.

42. Ruperto, N., Ravelli, A., Pistorio, A., Malattia, C., Cavuto, S., Gado-West, L., et al. (2001). Cross-cultural adaptation and psychometric evaluation of the Childhood Health Assessment Questionnaire (CHAQ) and the Child Health Questionnaire (CHQ) in 32 countries. Review of the general methodology. Clinical and Experimental Rheumatology, 4 Suppl 23(23), S1-S9.

43. Goldstein, H. (1995). Multilevel statistical models (2nd ed.). London: Edward Arnold.

44. Hirschhorn, J. N., \& Daly, M. J. (2005). Genome-wide association studies for common diseases and complex traits. Nature reviews. Genetics, 6, 95-108.

45. Hofman, A., Breteler, M. M., van Duijn, C. M., Krestin, G. P., Pols, H. A., Stricker, B. H., et al. (2007). The rotterdam study: Objectives and design update. European Journal of Epidemiology, 22, 819-829.

46. Sprangers, M. A. G., et al. (2010). Scientific imperatives, clinical implications, and theoretical underpinnings for the investigation of the relationship between genetic variables and patient-reported quality-of-life outcomes. Quality of Life Research. doi:10.1007/ s11136-010-9759-5.

47. Lubetkin, E. I., Jia, H., Franks, P., \& Gold, M. R. (2005). Relationship among sociodemographic factors, clinical conditions, and health-related quality of life: Examining the EQ-5D in the U.S. general population. Quality of Life Research, 14, 2187-2196.

48. Panepinto, J. A., O'Mahar, K. M., DeBaun, M. R., Loberiza, F. R., $\&$ Scott, J. P. (2005). Health-related quality of life in children with sickle cell disease: Child and parent perception. British Journal Haematology, 130(3), 437-444.

49. Houtzager, B. A., Grootenhuis, M. A., Caron, H. N., \& Last, B. F. (2005). Sibling self-report, parental proxies, and quality of life: the importance of multiple informants for siblings of a critically ill child. Pediatric Hematology and Oncology, 22(1), 25-40. 